\title{
PERCEPÇÃO DE FAMILIARES DE CRIANÇAS INTERNADAS SOBRE O PAPEL DOS CUIDADORES
}

\author{
PERCEPTION FROM HOSPITALIZED CHILDREN FAMILIES \\ ABOUT PROFESSIONAL PAPER
}

\section{LA PERCEPCIÓN DE LAS FIMILIAS DE LOS NIÑOS INTERNADOS REFERENTE AL PAPEL DE LOS PROFESIONALES}

\author{
Heloisa Beatriz Machado* \\ Maria Gorete de Souza** \\ Carolina Machado*** \\ Bárbara Rebello****
}

\footnotetext{
* Enfermeira. Mestre, Universidade do Vale do Itajaí - Centro de Ciências da Saúde.

** Enfermeira. Mestranda, Universidade do Vale do Itajaí - Hospital Universitário Pequeno Anjo.

*** Acadêmica do Curso de Medicina da Universidade do Vale do Itajaí - Centro de Ciências da Saúde.

**** Enfermeira da Maternidade e Hospital Dia Santa Luiza.
}

RESUMO. O Estatuto da Criança e do Adolescente garantiu o direito de permanência de um familiar com a criança/adolescente hospitalizado. A inserção da família no ambiente hospitalar originou novas demandas com as quais os profissionais precisam interagir, entre elas, pensar no cuidado incluindo a família. Os pais têm necessidades que precisam ser atendidas pela equipe de saúde, como: receber informação, apoio psicológico, atenção, repouso e alimentação. A equipe de saúde tem papel fundamental para suavizar os sentimentos negativos do cliente e promover uma relação mais positiva com a família. O estudo objetivou verificar a percepção do familiar acerca do papel dos profissionais que interagem com a criança durante a hospitalização. O estudo foi de natureza exploratória, realizado com 50 familiares de criança/adolescentes internados no Hospital Universitário Pequeno Anjo, no período de agosto a dezembro de 2004. Os dados foram obtidos pela aplicação de formulário elaborado a partir de referências bibliográficas sobre o tema. A maioria dos cuidadores foram mães, jovens e com baixa escolaridade. Conhecem os profissionais que atendem a criança e as atividades que realizam. O profissional que mais interagiu com a criança/adolescente foi o auxiliar de enfermagem e o médico, seguindo-se o enfermeiro. $O$ significado do ser cuidador tem relação com o papel de ser mãe e um bom cuidador é aquele que dá atenção e carinho aos clientes. Para o familiar, o profissional com o perfil de bom cuidador é o auxiliar de enfermagem e demonstram satisfação com o atendimento recebido no hospital.

PALAVRAS-CHAVE: assistência à criança; cuidador, papel profissional.

ABSTRACT. The Statute of the Child and the Adolescent guaranteed the right of permanence of a familiar one with the hospitalized adolescent/child. The insertion of the family in the hospital environment originated new demands with which the professionals need to interact, between them, to think about the care including the family. The parents have necessities that they need to be taken care of by the health team, as: to receive information, psychological support, attention, rest and feeding. The health team has basic paper to alleviate the feelings negative of the customer and to promote a more positive relation with the family. The study it objectified to verify the perception of the familiar one concerning the paper of the professionals who interact with the child during hospitalization. The study it was of exploratória nature, carried through with 50 familiar of crianca/adolescentes interned in the University Hospital Small Angel, in the period of August the December of 2004. The data had been gotten by the form application elaborated from bibliographical references on the subject. The majority of the cuidadores mothers had been, young and with low escolaridade. The professionals who take care of the child and the activities know that carry through. The professional who more interacted with criança/adolescente was the nurse aid and the doctor, following itself the nurse. The meaning of the cuidador being has relation with the paper of being mâe and a good cuidador is that one that gives to attention and affection to the customers. For the familiar one, the professional with the profile of good cuidador is the nurse aid and demonstrates satisfaction with the attendance received in the hospital.

KEYWORDS: assistance to the child; cuidador; professional paper.

RESUMEN. El estatuto del niño y del adolescente garantizó el derecho a la permanencia de un familiar con el niño/adolescente hospitalizado. La inserción de la familia en demandas originadas el ambiente del hospital las nuevas demandas con las cuales los profesionales necesitan interagir entre ellas, pensar el cuidado incluyendo la familia. Los padres tienen necesidades que necesitan ser suplidas por el equipo de salud, como: recibir información, ayuda psicologica, atención, el resto y la alimentación. El equipo de salud tiene un papel básico al aliviar las sensaciones negativas del cliente y al promover una relación positiva con la familia. Es un estudio explorado realizado con so familiars de niños/adolescentes internados en el Hospital Universitário Pequeno Anjo, para verificar la opinión de la familiar referente al papel de los profesionales que obran recíprocamente con el niño durante la hospitalización. El estudio estaba de naturaleza del exploratória, llevado a través con el familiar 50 de criança/adolescentes internado en el ángel pequeño del hospital de la universidad, en el período de agosto el diciembre de 2004. Los datos habían sido conseguidos por el uso de la forma elaboraron de referencias bibliográficas en el tema. La mayoría de los cuidadores sirve de madre había sido, los jóvenes y con el escolaridade bajo. Los profesionales que toman el cuidado del niño y de las actividades saben que lleva a través. El profesional a que más obraron recíprocamente con criança/adolescente era el asistente de la enfermera y el doctor, siguiéndose la enfermera. El significado del cuidador que es tiene relación con el papel de ser mâe y un buen cuidador es aquél que da a la atención y al afecto a los clientes. Para el familiar, el profesional con el perfil del buen cuidador es el asistente de la enfermera y demuestra la satisfacción con la atención recibida en el hospital.

PALABRAS-CLAVE: ayuda al niño; cuidador; papel profesional.

Recebido em: 20/10/2005

Aceito em: $21 / 11 / 2005$
Heloisa Beatriz Machado

Rua Uruguai, 458 - 88302-202 - Itajaí - SC

E-mail: heloisa@ccs.univali.br 


\section{INTRODUÇÃO}

Ser cliente em um ambiente de cuidados, seja ele hospitalar ou ambulatorial, pode ser potencialmente amedrontador, confuso e de certa forma desumano, pela quebra que provoca no cotidiano das pessoas, pelo medo do desconhecido e mesmo pela impessoalidade das relações ${ }^{1}$. Tais situações podem, de início, desequilibrar um adulto e, principalmente uma criança, cujo desenvolvimento e maturidade estão em construção. O Hospital Universitário Pequeno Anjo foi assumido pela Universidade do Vale do Itajaí em 2002 e vem, nestes dois anos, adequando sua área física, bastante antiga, às atuais necessidades e exigências da legislação sanitária, bem como, investindo em processo de educação permanente dos profissionais e de implantação de um programa de humanização que supere a simples permanência de um familiar junto à criança hospitalizada e a ampliação do horário de visitas.

Com esta perspectiva, a equipe de saúde tem um papel fundamental para suavizar os sentimentos negativos do cliente e promover uma relação mais positiva. Por vezes, o profissional parece esquecer que o seu agir "toca" a dignidade e a liberdade do outro. A rotina diária de trabalho facilita ao profissional "esconder-se" por detrás das rotinas, esquecendo o essencial de sua profissão: $o$ ato de cuidar ${ }^{2}$. Nesse momento deixa de ser profissional de saúde, passando a ser apenas um técnico, capaz de realizar tratamentos eficazes, negligenciando o cuidado. Muitas vezes, o profissional trata do corpo mas esquece a pessoa e seu mundo. No caso da criança o componente afetivo é tão ou mais importante do que o cuidado técnico, pois é capaz de transmitir-lhe segurança e algum sentido de normalidade.

Para minimizar os efeitos da separação, mesmo que temporária, entre pais e filhos é que o Estatuto da Criança e do Adolescente (ECA), garante a permanência em tempo integral de um dos pais ou responsável, nos casos de internação de crianças ou adolescentes ${ }^{3}$.

Com a implementação do ECA e a inserção da família no ambiente hospitalar, surgiram novas demandas com as quais os profissionais precisam interagir, entre elas, mudar o foco da atenção da doença para o cuidado e um cuidado que seja inclusivo, pensando na criança/adolescente e sua família. Os profissionais precisam entender que os pais também tem suas necessidades que precisam ser atendidas, eles precisam por exemplo de informação confiável sobre a situação do seu filho, tem necessidades físicas, repouso, alimentação, etc...) e emocionais (atenção, orientação, apoio psicológico) e precisam ser preparados para participar do cuidado durante a hospitalização e após a alta ${ }^{3}$.

O acolhimento e a troca de informações com a família, significa compartilhar as idéias e os cuidados, buscando incorporar as observações feitas pelos familiares no plano de cuidados da criança/ adolescente ${ }^{3}$.

Cuidar de uma forma expressiva e sincera, transmitindo apoio aos clientes e às suas famílias tem sido o desejo dos profissionais que caminham em busca de um cuidado mais humanizado e é também a meta do Programa de Humanização do Hospital Universitário Pequeno Anjo, o que motivou a realização desse trabalho.

\section{OBJETIVOS}

O estudo teve como objetivo verificar a percepção do familiar cuidador acerca do papel dos profissionais que interagem com a criança durante a hospitalização. Como objetivos específicos buscamos verificar a percepção do cuidador em relação às atividades desenvolvidas pela equipe, bem como identificar entre as atividades desenvolvidas as que mais caracterizam o papel do profissional como cuidador; verificar o profissional que mais interage com a criança e família, a percepção do familiar sobre a forma como o cuidado prestado e o cuidador interferem na recuperação da criança.

\section{METODOLOGIA}

Desenvolveu-se um estudo de cunho exploratório com abordagem quantitativa, que envolveu os todos 
os familiares acompanhantes de crianças internadas, no período de agosto a dezembro de 2004. O local do estudo foi o Hospital Universitário Pequeno Anjo, que atende crianças e adolescentes na faixa de zero a 14 anos de idade, sendo que predominam as internações na faixa etária de zero a 7 anos. O único critério de inclusão era aceitar participar da pesquisa, assinando o termo de consentimento livre e esclarecido. Desta população, obtivemos uma amostra de 50 cuidadores. A coleta de dados foi realizada pelas pesquisadoras no período vespertino e aos sábados e foi aplicado um formulário elaborado a partir da literatura, buscando identificar quem é o cuidador, como o familiar identifica esse cuidador, vínculos estabelecidos e o papel esperado em relação ao tratamento da criança. Os dados obtidos foram tabulados manualmente, apresentados através de distribuição de freqüência absoluta e relativa $e$ comparados ao referencial teórico utilizado no estudo, conforme demonstrado abaixo.

\section{RESULTADOS E DISCUSSÕES}

Organizou-se uma síntese dos principais resultados e quanto ao perfil dos familiares cuidadores, verificou-se predominância de mulheres com o primeiro grau incompleto e procedentes do município de Itajaí - SC. A maioria das mães eram bastante jovens, na faixa etária entre 15 a 30 anos. Entre as mulheres, em grande parte a mãe é que cuidava e acompanhava a criança, embora tenha ocorrido também a presença da avó, da tia, entre outras. Quando o cuidador era homem, quase a totalidade era representada pela figura do pai. Tal resultado era esperado e também foi encontrado em outros estudos ${ }^{3 ; 4}$ estando relacionado com 0 fato do pai ter emprego fixo e a mãe, na sua maioria relatar como ocupação cuidar da casa e dos filhos. Mesmo quando a mãe trabalha, é mais freqüente a mulher faltar ao trabalho do que o homem, pois o cuidado tem sido entendido há muitos anos como responsabilidade feminina. O cuidado tem sido assumido pela mulher, que responsabiliza-se pela atenção à saúde de toda a família e dela mesma.
Os dados obtidos no estudo de Sabatés e Borba $^{3}$ revelaram que a mãe foi a pessoa que mais permaneceu com o filho hospitalizado, exerciam atividades do lar, escolaridade entre a $1^{\mathrm{a}}$ e a $7^{\mathrm{a}}$ série do ensino fundamental, com idade entre 21 e 30 anos, dados semelhantes aos encontrados nesse estudo.

Alguns autores ${ }^{4 ; 5}$ relatam que a presença da mãe tem a preferência da criança, o que está relacionado com vínculo afetivo e paciência com as manifestações de dor e de medo da criança como: irritação, choro e inquietude. A presença da mãe geralmente acalma e transmite segurança à criança hospitalizada, principalmente para as de menor idade, que no seu cotidiano convivem maior tempo com a mãe. As mães apresentam elevada capacidade de dedicarem-se aos seus filhos nos momentos em que precisam de toda a atenção, suprindo suas necessidades de alimentação, higiene, acalento e amor ${ }^{3}$.

Na figura 01, a seguir, verifica-se que $88,57 \%$ dos participantes da pesquisa tiveram contato com o médico, e o mesmo percentual com os auxiliares de enfermagem, sendo ambos os profissionais mais citados pelos cuidadores. Ressalta-se também que $80 \%$ dos participantes, além de conhecer o médico e o auxiliar de enfermagem, conhece também o enfermeiro, embora não o reconheça pelo nome, como ocorre com o médico. O psicólogo, o nutricionista e o fisioterapêuta foram citados por $34,28 \%$ das mães e o assistente social por 51,42\%.

O assistente social ocupou a terceira posição em termos de percentuais, mas acreditamos, pela observação diária durante a realização das entrevistas, que este profissional tem um papel muito importante de suporte à mãe e à família, sendo procurado e conhecido por muitas dessas famílias, buscando auxílio para transporte, alimentação, roupas para a criança ou adolescente, local para pernoite, orientações, entre outras necessidades que precisam ser atendidas para que a mãe possa realmente dar atenção à criança e ou adolescente quando hospitalizados. No hospital esse profissional tem uma 
atribuição fundamental, que é a de orientar a criança, adolescente e família, sobre seus direitos e deveres, as rotinas do hospital, benefícios e encaminhá-los aos programas existentes, como por exemplo, para as atividades do programa de humanização do hospital.

Figura 1 - Distribuição dos profissionais que mantiveram contato com a criança, segundo a percepção do cuidador

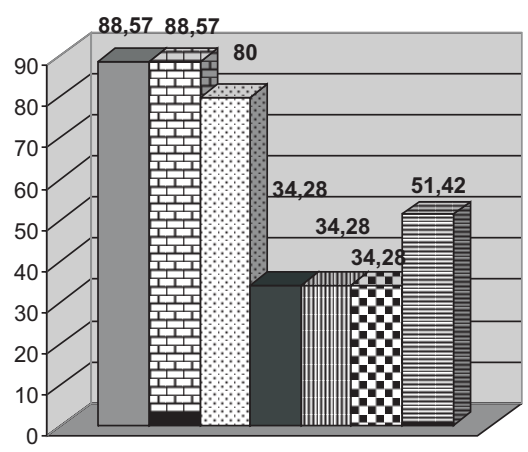

$\square$ Médicos

ÐAux. Enfermagem $\checkmark$ conhece enfermeiro

$\square$ Psicólogos

四 Nutricionista

四Fisioterapeutas

目Assitênte socia

Além de relatar que conhece o profissional que tem contato com a criança e ou adolescente, as mães demonstraram conhecer algumas das atividades desenvolvidas por esses profissionais, conforme demonstrado no quadro 01, a seguir.

Quadro 1- Atividades desenvolvidas segundo a categoria profissional

\begin{tabular}{|l|l|}
\hline \multicolumn{1}{|c|}{ Profissional } & \multicolumn{1}{c|}{ Atividade } \\
\hline Assistente Social & $\begin{array}{l}\text { Cuida da parte social, entrega } \\
\text { passes de transporte coletivo e } \\
\text { ajuda famílias mais carentes }\end{array}$ \\
\hline Médico & $\begin{array}{l}\text { Examina e atende a criança no } \\
\text { quarto }\end{array}$ \\
\hline Fisioterapeuta & Faz massagem \\
\hline Nutricionista & $\begin{array}{l}\text { Orienta quanto ao que a criança } \\
\text { pode ou não comer }\end{array}$ \\
\hline Psicóloga & $\begin{array}{l}\text { Conversa com a família para } \\
\text { saber do caso da criança }\end{array}$ \\
\hline Enfermeiro & $\begin{array}{l}\text { Cuida dos remédios e dá atenção } \\
\text { aos pacientes }\end{array}$ \\
\hline Auxiliar de Enfermagem & $\begin{array}{l}\text { Administram os medicamentos, } \\
\text { cuidam do soro (fluídoterapia), } \\
\text { verificam sinais vitais e auxiliam o } \\
\text { enfermeiro. }\end{array}$ \\
\hline
\end{tabular}

Os resultados obtidos mostraram que o familiar reconhece algumas atividades desenvolvidas pelos profissionais de saúde que prestam assistência à criança, separando-as por categoria. Aqueles que tiveram contato com a assistente social referem que esta profissional cuida da parte social, entrega passes de transporte coletivo e ajuda as famílias mais carentes. O médico examina e atende a criança no quarto, a fisioterapeuta faz massagem e a nutricionista orienta quanto ao que a criança pode ou não pode comer. Referem ainda que a psicóloga conversa com a família para saber do caso da criança.

Quanto à equipe de enfermagem, para a família o enfermeiro é aquele profissional que cuida dos remédios e que dá atenção aos pacientes. Alguns familiares colocam que é o profissional que tira as dúvidas da família. Na percepção dos cuidadores, os auxiliares de enfermagem são aqueles que administram os medicamentos, cuidam do soro (fluidoterapia), verificam os sinais vitais e auxiliam o enfermeiro em suas atividades.

Tais percepções estão carregadas pela vivência diária numa unidade de internação e refletem o que perpassa para o senso comum em relação ao papel profissional, a busca de informações e o diagnóstico da situação da criança/adolescente parece tradicionalmente vinculados a figura do médico, e o fato de providenciar os cuidados diários para garantir a terapêutica, tem sido vinculado ao trabalho do auxiliar de enfermagem. Esta situação também foi encontrada por Sigano, Sigaud e Rezende ${ }^{5}$ no qual os pais identificaram as auxiliares como as profissionais que arrumam e dão banho na criança, que trazem o medicamento na hora certa e aplicam os fármacos necessários para o êxito do tratamento.

Chama a atenção o fato do familiar identificar o enfermeiro como o profissional que tira as dúvidas do famíliar e dá atenção não só para a criança/ adolescente, mas para a família. Em seu estudo, Sigano, Siagau e Rezende ${ }^{5}$ verificaram que a enfermeira é a profissional que "cuida de tudo", designação entendida como ligada a atividade de hospedagem que as autoras consideram como uma representação social do trabalho de enfermagem como extensão do trabalho doméstico. Nesse estudo as mães também perceberam a enfermeira como a profissional "que informa, orienta e apóia" e sentiramse incluídas nesse cuidado. 
Em outro estudo conduzido por Sabatés e Borba $^{3}$ em São Paulo, as autoras identificaram que as enfermeiras foram as profissionais que mais informaram os pais sobre os motivos dos procedimentos realizados em seus filhos e o motivo da internação, o que corrobora com os dados obtidos. As autoras consideram que a informação é essencial para uma interação adequada entre a enfermeira e a família da criança hospitalizada, constituindo passo fundamental para a inclusão da família no cuidado.

Figura 2 - Percepção do cuidador quanto ao profissional que mais interagiu com a criança/adolescente

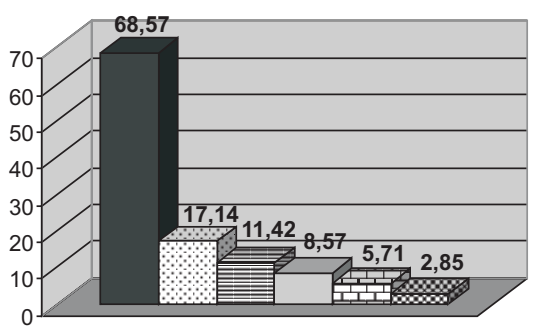

$\square$ auxiliar enfermeiro

Đenfermeiro

目psicologo

$\square$ fisioterapeuta

Đmedico

⿴囗十 pedagogo

A figura 02 acima, demonstra que o profissional que mais interagiu com a criança/adolescente também foi o auxiliar de enfermagem $(68,57 \%)$, seguindo-se o enfermeiro $(17,14 \%)$ e o psicólogo $(11,42 \%)$. fisioterapeuta foi citado por 8,57\% dos participantes, o médico por $5,71 \%$ e o pedagogo por $2,85 \%$.

Mais uma vez, pela natureza de suas atribuições, o auxiliar de enfermagem foi citado como o profissional que mais interagiu com a criança/adolescente durante o período da internação. As mães assim os reconhecem, porque passam a maior parte do tempo nos quartos organizando e cuidando, situação relatada como "permanência prolongada nas enfermarias junto às crianças" 5 .

Quanto ao psicólogo, este hospital desenvolve um estágio de psicologia hospitalar, com ênfase ao preparo pré-operatório e às crianças/adolescentes com distúrbios considerados prioritários para a psicologia clínica. Desta forma, o psicólogo bem como os acadêmicos do curso de psicologia estão diariamente no hospital, realizando através do contato com a família, uma triagem para proceder ao atendimento.
Vale destacar que o assistente social, que ocupava o terceiro lugar na citação quanto a ser conhecido pela família, neste quesito não foi citado. Tal fato pode estar relacionado ao entendimento do familiar de que a pergunta referia-se a uma interação mais efetiva com a criança e que a assistente social do hospital tem uma relação mais direta com a mãe ou familiar.

Em relação ao cuidado recebido, 91,42\% sentem-se apoiados pela equipe que assiste a criança/adolescente, independente da categoria profissional. Esse dado foi referido pelos familiares em relação estrita a capacidade técnica das equipes e se revela num dado positivo, pois competência técnica é uma condição essencial para que o cuidado em sua concepção mais ampla seja implementado.

Figura 3 - Distribuição dos participantes quanto ao significado do termo "ser cuidador"

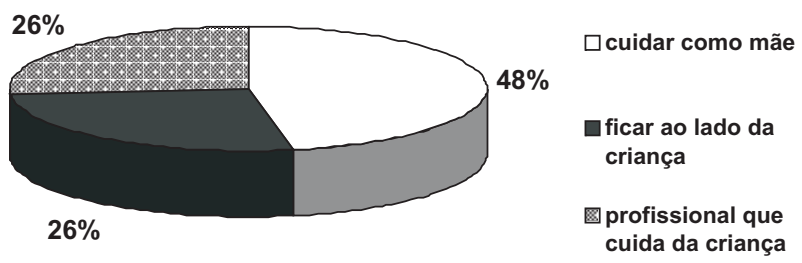

Quanto ao significado do que é ser um cuidador (fig. 03), os participantes entendem que é o cuidado como o executado pela mãe $(45,71 \%)$, seguindo-se o profissional que fica sempre ao lado da criança $(25,71 \%)$ e aquele que cuida da criança $(25,71 \%)$. Percebe-se que em todas as percepções relatadas pelos familiares, manifesta-se o desejo que o profissional cuidasse da criança como se fosse a mãe. Na sua percepção, as crianças precisam ser bem tratadas, cuidadas com carinho e amor, com brincadeiras, docilidade e afeto, atributos vinculados à atividades tidas como femininas ${ }^{5}$.

Dada a natureza da compreensão sobre o que é ser cuidador, concepção expressada como ligada à atividade feminina e ao papel da mãe (fig. 03), na percepção dos familiares que participaram da pesquisa, o profissional que melhor desempenha esse 
papel de cuidador é o auxiliar de enfermagem $(65,71 \%)$, seguindo-se o enfermeiro $(31,42 \%)$, pelos motivos relatados anteriormente e o médico $(17,14 \%)$. O nutricionista foi citado por 8,57\%, o fisioterapeuta por 5,71\%, o assistente social e o psicólogo por $2,85 \%$ dos familiares participantes. Os achados deixam claro que o cuidador, na percepção dos familiares é o profissional de nível médio e não o profissional de nível superior (fig.04).

Figura 4- Percepção dos familiares quanto ao profissional que melhor desempenha o papel de cuidador

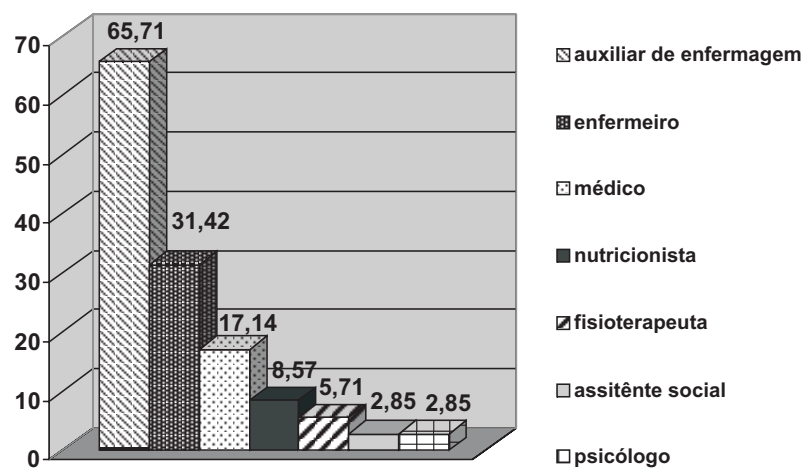

Para os familiares que participaram do estudo, o perfil de um profissional caracterizado como bom cuidador implica em ser atencioso com a criança e a família $(45,71 \%)$, ser carinhoso com a criança $(31,42 \%)$, ser paciente $(20 \%)$, ser responsável $(17,14 \%)$ e ser cuidadoso $(5,71 \%)$, conforme demonstrado na figura 05 abaixo.

Figura 5- Percepção dos respondentes quanto ao perfil do bom cuidador

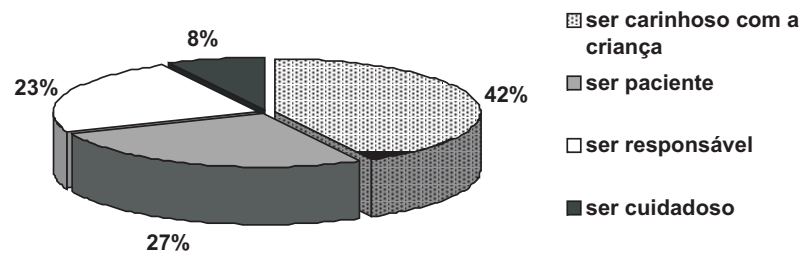

Já com relação à satisfação com o cuidado recebido no hospital (fig. 06), 48,57\% dos familiares entrevistadas mostraram-se totalmente satisfeitos com o cuidado recebido pela criança/adolescente, 40,93\% estão parcialmente satisfeitos, sendo que $14,28 \%$ alegaram que para um cuidado mais humanizado deveria ter um banheiro exclusivo para as mães no andar da unidade de internação, 12,37\% solicitaram melhorias nas acomodações para o acompanhante, $8,57 \%$ gostariam de receber mais orientações por parte do enfermeiro, 5,71\% solicitaram a inclusão de um horário de visita para os irmãos.Verificou-se um percentual de insatisfação de 10,50\%, sendo que apenas $2,85 \%$ destes, relataram que precisa melhorar o acolhimento da família.

Figura 6- Distribuição dos respondentes quanto a satisfação com o cuidado recebidol

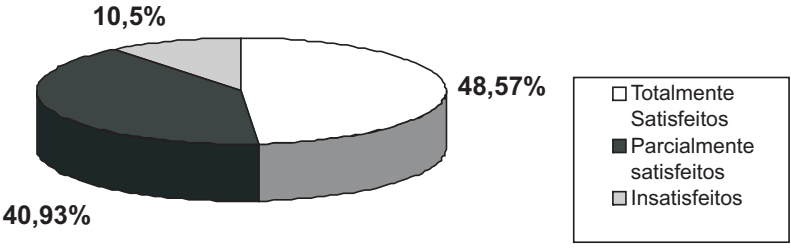

Os resultados obtidos demonstram que 0 conforto e a inclusão da família (pai, mãe e irmãos) é reconhecido como importante para promover conforto, dar carinho e atenção a criança/adolescente e a ampliação do horário de visitas como fator de humanização. Como o horário no Hospital já é ampliado nos períodos da tarde e noite, os pais sugerem a possibilidade de que os irmãos possam se visitar. As solicitações relacionadas à área física e acomodações justificam-se pelo fato do hospital estar passando por período de reforma, prejudicando ainda mais a área que já é pequena e não possibilita nesse momento benfeitorias. Segundo a direção do hospital quando terminadas as obras nas unidades $A$ e B do hospital, todos os quartos terão banheiro privativo e cadeira confortável para cada um dos leitos.

Chama atenção o fato de que $8,57 \%$ dos familiares reconhece a importância do papel do enfermeiro e gostaria de receber mais informações desse profissional, o que implica em atenção e cuidado.

\section{CONSIDERAÇÕES FINAIS}

Os resultados apresentados permitem destacar que os familiares ao expressar suas percepções, separam o conceito de cuidado em dois elementos fundamentais: o cuidado entendido como a realização 
das técnicas e atividades que são essenciais para a recuperação física da criança e o cuidado na perspectiva de dar o suporte emocional de que a criança e a família necessitam para enfrentar a hospitalização, verbalizados como escuta, atenção, carinho e paciência. Pesquisadores como Costenaro, Daros e Arruda ${ }^{6}$ Gomes; Lunardi Filho ${ }^{7}$ e Passos ${ }^{4}$, ressaltam que muitas vezes a prática do enfermeiro e sua equipe se direciona essencialmente para o cuidado técnico, distanciando-se da dimensão humana de seus clientes e, portanto, relegando a segundo plano suas demais necessidades. A literatura deixa evidente que pacientes e suas famílias esperam dos profissionais uma atuação segura, mas com manifestação de carinho, paciência e interação nos relacionamentos, o que em muitas instituições ainda parece não ocorrer ${ }^{4}$. É animador e ao mesmo tempo desafiador reconhecer que a família estabelece mais vínculos com a equipe de enfermagem e com 0 enfermeiro, que tem expectativas em relação ao papel desses profissionais e que também quer ser acolhida e cuidada ${ }^{8}$.

Conhecer a percepção de crianças e familiares acerca do papel e o que esperam do profissional de saúde pode representar uma forma de contribuir para se prestar um cuidado integral, humanizado e que atenda as necessidades dos clientes, ajudando-os a superar alguns estressores ligados a hospitalização, tais como: as proibições que a criança vivencia durante a doença e a internação, solidão, saudade, ausência de outros membros da família, medo da dor e do desconhecido, do tratamento e dos procedimentos invasivos, entre outros.

Neste sentido ressalta-se que para chegar ao cuidado humanizado pretendido é preciso ouvir a criança em sua própria linguagem, respeitando seu modo peculiar de ver o mundo, além de exercitar o cuidado da família, pois sabe-se hoje que a família também adoece junto com seus membros, mas que quando recebe o suporte de que necessita é também um fator que contribui para a recuperação da criança/ adolescente. Destaca-se ainda que é fundamental o cuidado despendido pela equipe de enfermagem, uma vez que esses profissionais são apontados como os que mais interagem com a criança e família, sendo sua atuação fundamental para a inclusão da família e a promoção da saúde da criança.

\section{REFERÊNCIAS}

1 Capparelli ABF. A doença, a criança e sua família. Ped Mod 1998; 34:59.

2 Boff L. Saber cuidar: ética do humano, compaixão pela terra. São Paulo: Vozes; 1999.

3 Sabatés AL, Borba RIH. As informações recebidas pelos pais durante a internação. Rev Latino-Am Enferm 2005; 13(6): 968-73.

4 Passos FM. Fatores que facilitam e dificultam o cuidado humanizado na percepção das mães que vivenciando uma internação pediátrica junto de seus filhos. [monografia]. Itajaí (SC): Universidade do Vale do Itajaí; 2002.

5 Sugano AS, Sigaud CHS, Rezende MA. A enfermeira e a equipe de enfermagem - segundo mães acompanhantes. Rev Latino-Am Enferm 2003; 11(5): 601-7.

6 Costenaro RGS, Daros A, Arruda EN. O cuidado na perspectiva do acompanhante de crianças e adolescentes hospitalizados. Rev Enferm Escola Anna Nery 1998; 2(2): 137-41.

7 Gomes GC, Lunardi Filho WD. A família uma unidade que se cuida, uma unidade a ser cuidada. Texto e Contexto Enferm 2000; 9(2): 28-8.

8 Silva LF. et al. Cuidado de enfermagem: o sentido para enfermeiros e pacientes. Rev Bras Enferm 2001; 54(4): 578-88. 